\title{
Nanofabrication with Ion Beams Visualized in the Environmental Scanning Electron Microscope
}

\author{
John Mansfield*, Rachel Goldman**, Richard Laine and Max Shtein** \\ *University of Michigan North Campus Electron Microbeam Analysis Laboratory, 417 SRB, 2455 \\ Hayward, Ann Arbor, MI 48109-2143, USA \\ ** University of Michigan Department of Materials Science and Engineering, 2300 Hayward Street, \\ Ann Arbor, MI 48109-2136, USA
}

Environmental scanning electron microscopes (ESEMs) and variable pressure scanning electron Microscopes (VPSEMs) are now common instruments, FEI Company offer several models of ESEM and JEOL, Hitachi and Zeiss each offer VPSEMs. In addition, FEI and Zeiss have been offering dual-beam focused ion beam workstations (FIBs) for a number of years. The blending of the two technologies, a dual-beam ESEM/VPSEM and FIB is a comparatively recent innovation and such an instrument has been installed in the North Campus Electron Microbeam Analysis laboratory NC EMAL) at the University of Michigan. The system is an FEI Company Quanta 200 3D, the electron optical column is tungsten-filament based and is capable of operating in high vacuum $\left(<10^{-3} \mathrm{~Pa}\right)$, variable pressure (up to $\sim 70 \mathrm{~Pa}$ ) and ESEM (up to $\sim 3000 \mathrm{~Pa}$ ) modes. The instrument is fitted with a factory-standard Pt gas injector for platinum metal deposition and a factory-standard solid-state back scatter detector. A Peltier stage and a hot stage are also available. A Noran UTW XEDS detector with 4Pi Analysis acquisition hardware and software has been attached to the system for chemical analysis.

We have determined that this ESEM/FIB will make an excellent platform for both processing and observing integrated inorganic-organic nanostructures and nanocomposites. For example, Shtein and Goldman plan to explore the formation of hybrid organic/inorganic systems for photovoltaic devices for renewable energy applications and Goldman and Mansfield will explore the real-time formation of semiconductor nanopillars, upon which thin films of silsesquioxanes will be selectively vapor deposited by Laine and Shtein. Goldman and Mansfield will also explore the fabrication of electroluminescent nanopillar p-n junctions, to be combined with vaporphase deposited "cubes" (Laine and Shtein), for high-resolution full-color displays. To perform the materials depositions inside the ESEM/FIB, two additional deposition and gas injector systems are being installed on the instrument. One, for the deposition of organic and silicon based molecules, will be a modified FEI gas injector and the other will be a gas micro-injector for ammonia.

The first step in the nanocomposite and nanostructure fabrication will make use of the gallium droplet formation that occurs when gallium arsenide (or any other Ga containing semiconductor) is subjected to extended exposure in the FIB. The Ga droplets form after the substrate is supersaturated with $\mathrm{Ga}$, an effect that is typically undesirable when working with gallium containing compounds. Size and site-controlled Ga nanodots will be seeded by FIB implantation. An example of Ga FIB-fabricated hole arrays is shown in Fig. 2(a). These holes are highly ordered and have nearly uniform sizes and shapes. By controlling the ion beam energy, current, and size, hole arrays with various sizes, depths, and periodicities may be produced. Scanning the ion beam over the patterned areas allows the formation of Ga dots at the centers of the holes, which results in ordered arrays of nearly uniform sized Ga dots [Fig. 2(b)]. These dots will be annealed in the ESEM/FIB, and vapor-liquid-solid VLS growth of nanopillars will be initiated by exposure to a gaseous flux using in-situ injection of various gaseous and sublimated solid sources, including ammonia and 
silsesquioxanes. Self-assembled GaN quantum dots have been grown on $6 \mathrm{H}-\mathrm{SiC}$ substrates via the formation and nitridation of Ga liquid droplets [1]. Thin films of silsesquioxanes and other organic molecules will be selectively attached to the semiconductor nanostructures by vapor-phase ionassisted deposition and cured with ion and/or electron beams.

\section{References}

1. C. W. Hu, A. Bell, F. A. Ponce, et al., "Growth of self-assembled GaN quantum dots via the vaporliquid-solid mechanism", Applied Physics Letters 81, 3236 (2002

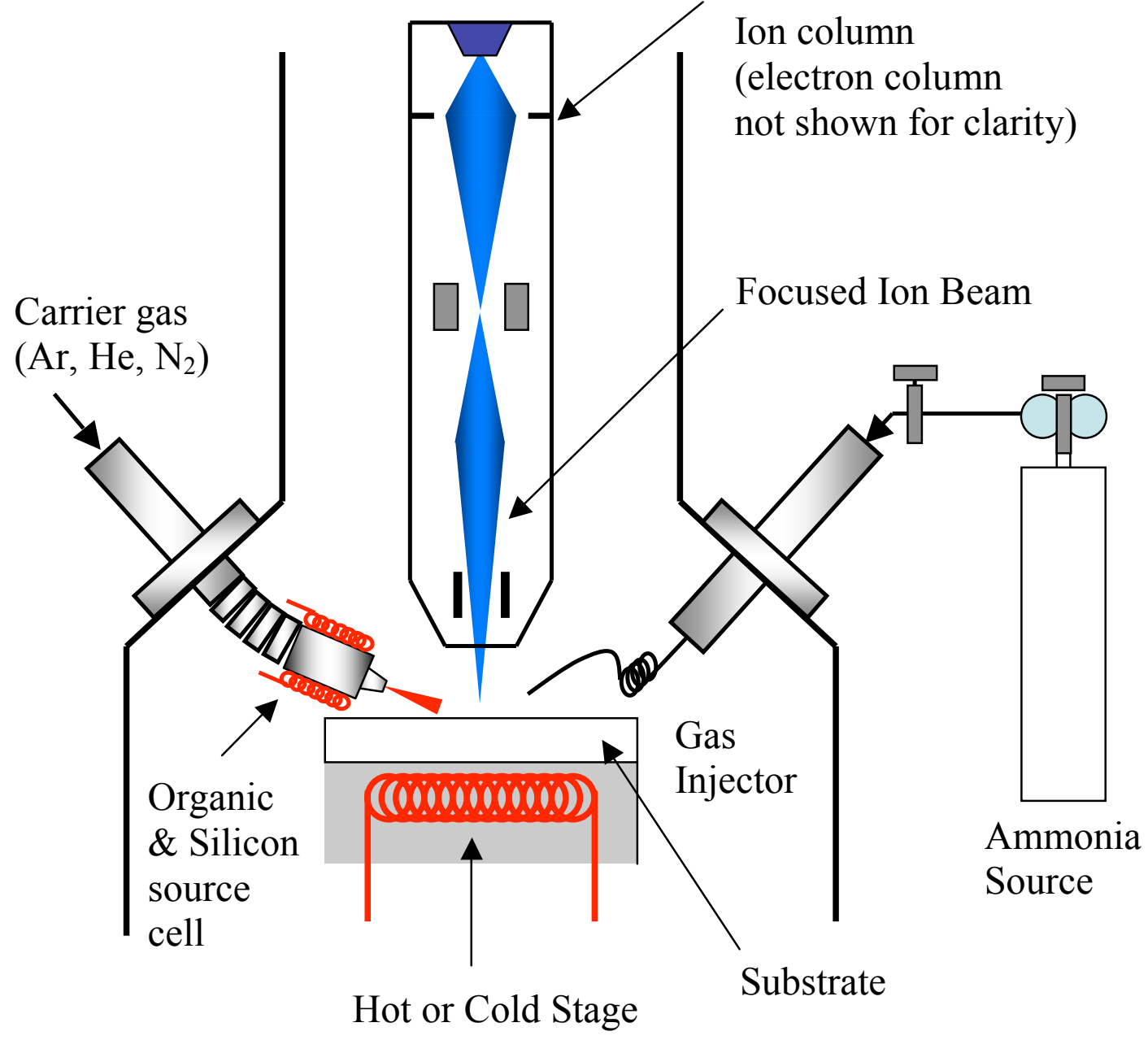

Fig. 1 Schematic Diagram of the ESEM/FIB with the additional fabrication sources.
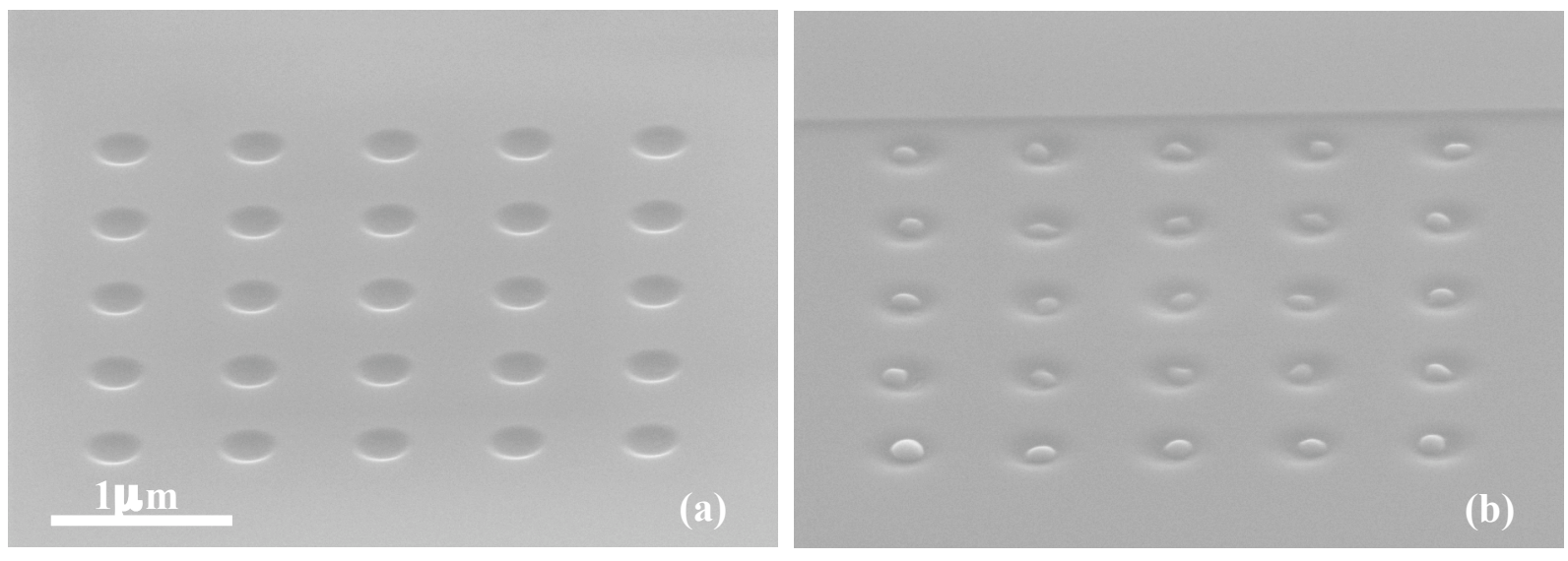

Fig. 2 (a) FIB-fabricated arrays of holes in GaAs (b) Ga droplets formed at each hole. 